\title{
Bearing monitoring in variable speed regime using normalized indicators
}

\author{
Khalid Ait Sghir ${ }^{1, a}$, Olivier Cousinard ${ }^{1,2}$, Fabrice Bolaers $^{1}$, Philippe Estocq $^{1}$ \\ AND Jean PaUl Dron ${ }^{1}$ \\ ${ }^{1}$ Groupe de Recherche en Science Pour l'Ingénieur, Université de Reims Champagne Ardenne, UFR Sciences \\ Exactes et Naturelles, Moulin de la Housse, BP 1039, 51687 Reims Cedex 2, France \\ 2 Société Altéad Industrie Est, 11 rue du colonel Charbonneaux, 51100 Reims, France
}

Received 9 March 2015, Accepted 12 October 2015

\begin{abstract}
In this paper, the diagnostic of rolling element bearings in variable regime is addressed. In the nonstationary regime, permanent changes of operating system parameters (load and speed) prevent fault diagnostic with classical tools (RMS value and spectral indicator). To overcome this problem, two new indicators are proposed: $R M S$ value and spectral indicator normalized by the active power. To do that, the vibration signal was acquired simultaneously with the current and voltage signals, and then these signals were divided into segments. For each segment, the $R M S$ value (respectively the spectral indicator) and the power active were calculated, then the $R M S$ was divided by the active power. An analytical model of vibration signal was given and a simulation model signal was used to investigate the proposed indicators. For the experimental validation, a test rig was performed to extract signals (vibration, current and voltage signals) at different degradation states of bearings in a variable speed regime. The thrust bearings (SKF 51207) were used with different states of degradation: free fault and faults created with different sizes. Several operating parameters were considered: variable speed (50-950 rpm) and different loads. The experimental tests showed a linear relationship between the classical $R M S$ value and the active power. The results showed a correlation between the proposed indicators and the bearings state in variable regime. Thus, the proposed indicators are very simple and may be implemented in real time for large industrial applications.
\end{abstract}

Key words: Vibration analysis / variable speed regime / classical indicators / active power / thrust bearings

\section{Introduction}

Rolling element bearings are considered among the most critical components in industrial machines. The main mode of failing of those components is spalling of the races or the rolling elements. This failure is caused by fatigue cracks which, under loading, begin below the surface of the metal and progress to the surface where they cause material to break loose at the contact area [1]. The failure of a rolling element bearing may have a catastrophic consequence on the machine if it is not detected and not followed up.

Several methods attempt to detect such localized defect at its incipient stage in order to prevent long-term breakdowns or in some cases possible catastrophic failures. Among those, vibration analysis has proven efficiency for the detection and diagnosis of bearing defects. When a defect is present, there is a significant increase

\footnotetext{
${ }^{a}$ Corresponding author: aitsrir@yahoo.com
}

in the vibration level. The main objective of vibration analysis consists in establishing and analyzing a robust indicator that can detect and quantify an incipient fault. In the literature, the majority of the indicators for bearing condition monitoring have been proposed under a steadystate regime (load and speed constant). The most used are the classical indicators, such as time indicators $[2,3]$ ( $R M S$ value, kurtosis, etc.) or spectral indicators $[4,5]$. Envelope detection [6] is a strong signal processing technique that has been widely used and its success has been demonstrated $[7,8]$. Time-frequency analysis is an effective tool for analyzing the behavior of transient signals [9]. Other signal processing tools have been proposed recently; for example, the cyclostationary tools, based on modelling vibration signal as a cyclostationary process, have been proposed and have demonstrated the effectiveness for bearing fault detection $[10,11]$.

Nevertheless, the diagnosis of rolling element bearing is more complex when the operating parameters of 
systems (speed, load) are variable. Under these nonstationary operations, the amplitude and frequency components change permanently. This affects clearly the classical diagnostic tools which cannot detect a potential fault in nonstationary condition. Several methods are proposed in the literature to treat these issues in monitoring condition. In reference [12], it is found that the classical spectral indicator is linear with the operating condition. This linearity forms a slope which is used as parameter for monitoring the planetary gearbox used in bucket wheel excavators. In reference [13], a method is proposed based on time-frequency analysis techniques combined with the automatic feature extraction method and fuzzy inference in order to automatically diagnose bearing faults in a variable speed regime. In reference [14], a statistical approach is proposed where the signal is divided into segments where speed is considered constant, then the mean and covariance matrices of the features vector are calculated, and the interpolation is made by the ranges of speed bins. A classification by a statistical approach is then applied in order to diagnose a gearbox running under variable speed. In reference [15], a normalization of the $R M S$ value by speed is proposed for diagnosing thrust bearings in a variable regime. In reference [16], a statistical diagnosis algorithm based on the significance level ( $p$-value) is developed and applied on a test-bed, composed of parallel gearbox and a planetary gearbox, working under a large variability of speed and load conditions.

In this study, a method is proposed to remove the speed variation effect on the RMS indicator and the spectral indicator. This is done with the normalization of those classical indicators by the active power. The paper is organized as follows: Section 2 presents the proposed methods; Section 3 presents the application of normalized $R M S$ to simulation signals; Section 4 outlines the experiments carried out; and Section 5 gives the result analyses before finishing with a conclusion.

\section{Proposed indicators}

\subsection{Signal vibration model}

Whenever a rolling element encounters a local defect (see Fig. 1a), a shock is generated that excites high frequency resonances of the whole structure between the bearing and the response transducer. This shock caused by the impact force of the bearing element on the defect is periodic and its shape is a Dirac with finite power (Fig. 1b). This Dirac is a result of the triangle, rectangle or parabolic form [17]. Several studies have developed analytical models of the impact force. In reference [18], a dynamic model is developed which gives the relationship between the impact force magnitude and a linear function of the rotational speed of inner race, the bearing geometry and the defect size. The model of the impact force $F_{i}$ given in reference [19] is considered in this work and is presented by the following equation:

$$
F_{i}=C_{1} v_{i} Q d e f
$$

where $C_{1}$ is a variable that depends on the bearing geometry [19]. $v_{i}$ is the rotating speed of the inner race (rpm); $Q$ is the load applied to the bearing $(\mathrm{N})$; def is the defect size $(\mathrm{mm})$.

On other hand, the vibration signal $x(n)$ captured by the accelerometer is modeled by the convolution product between the impact force $F_{i}(n)$ and the impulse response $h(n)$ of the system formed by the rolling element-outer race-shaft (Fig. 1a). This system is assumed to be linear time-invariant. It is also assumed that the dynamic behavior of the machine does not contain resonances that may amplify vibration measurements. The signal $x(n)$ can be written as:

$$
x(n)=E_{i}(n) \otimes h(n)+b(n)
$$

with $E_{i}(n)=\sum_{k=-\infty}^{\infty} F_{i}^{k} . \delta(n-k T)$ as a sequence of the impact forces; $b(n)$ as Gaussian white noise which represents the other vibration sources; and $\otimes$ as the convolution operation.

$$
x(n)=\sum_{k=-\infty}^{\infty} F_{i}^{k} h(n-k T)+b(n)
$$

Equation (3) gives the relationship between the impact force $F_{i}$ and the acceleration signal $x(n)$. In the following, we will present the active power and the normalized tools (RMS value and spectral indicator).

\subsection{Active power}

The active power is estimated by the following formula:

$$
P_{A}=\sqrt{3} U I \cos \varphi
$$

where $U$ is the $R M S$ value of the voltage (volts) between two phases of the supply of the three-phase motor, $I$ is the $R M S$ value of the current (amperes) passed through a phase, $\cos \phi$ is the power factor and for the brushless motor is equal to $1(\cos \phi=1) . P_{A}$ is the power active and is in watts. The active power can be written also by this equation:

$$
P_{A}=\frac{T_{u} \Omega}{n}
$$

with $n$ as the motor efficiency, $T_{u}$ as the output torque and $\Omega$ as the rotor revolution speed. From the relationship (5) one can see clearly that the active power is linearly related to the speed rotation $\Omega$ of the rotating shaft and the output torque which depends only on the applied load.

\subsection{RMS normalized by active power}

The $R M S$ value is an indicator, commonly used in the industrial area, which measures the level of static redressed energy of a vibration signal. The formula for its estimation is as follow:

$$
R M S=\sqrt{\sum_{n=1}^{N} \frac{(x(n))^{2}}{N}}
$$




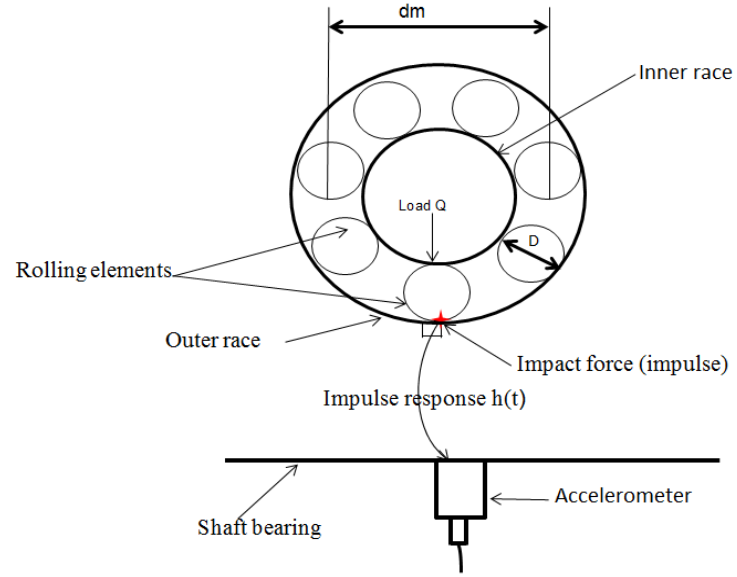

(a)

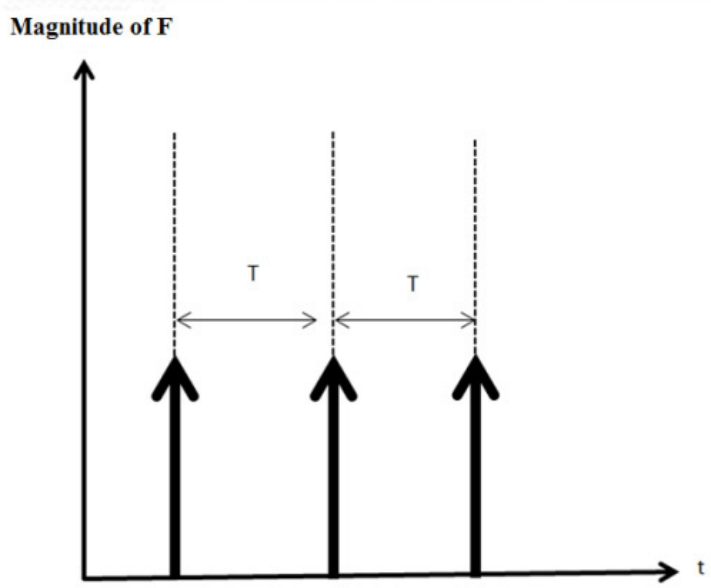

(b)

Fig. 1. (a) Modeling shock impact of the rolling element on a spalling defect. (b) Sequence of impact forces.

where $N$ is the number of samples and $x(n)$ is the sample of vibration signal at time $n$. The force impact, caused by the defect presence, increases the $R M S$ value.

For a number of samples $N$, on which the $R M S$ value is computed, it is assumed that the amplitudes of the impulses $E_{i}(n)$ are constant. The $R M S$ equation of the signal $x(n)$ is given by:

$$
R M S=\sqrt{\sum_{n=1}^{N} \frac{\left(F_{i} \sum_{k=-\infty}^{\infty} h(n-k T)\right)^{2}}{N}}+R M S_{0}
$$

with $R M S_{0}$ as the $R M S$ value of $b(n)$ ( $R M S$ in the free fault case).

Let $C_{2}=\sqrt{\sum_{n=1}^{N} \frac{\sum_{k=-\infty}^{\infty} h(n-k T)^{2}}{N}}$, a coefficient which is related to the impulse response $h(n)$ and which depends only on the system properties, the transmission path between the point of impact and the accelerometer sensor and the number of the shock at interval $N$ which is neglected. The value of $R M S$ is then:

$$
R M S=F_{i} C_{2}+R M S_{0}=C_{1} C_{2} Q v_{i} d e f+R M S_{0}
$$

Equation (8) provides that the $R M S$ value of the signal $x(n)$ depends linearly on the following: the load $Q$, the $C_{1}$ which depends to the bearing geometry, the coefficient $C_{2}$ which depends to the impulse response $h(n)$, the size of defect def, and the speed rotation of the inner race $v_{i}$.

The proposed method consists in normalization of the $R M S$ value by the active power. The algorithm to do that is simple and is as follows: the first step is acquiring the vibration signal at the same time as the current and voltage signals. Then all signals are segmented into segments of $\mathrm{N}$ points on which the signal is considered stationary. Then for each segment of a vibration signal and its corresponding voltage and current signals, we compute the $R M S$ value and the active power, then divide the $R M S$ value by the active power. This allows eliminating the influences of speed rotation and the load from Equation (8). Therefore, the normalized $R M S$ value is used for the diagnosis which will depend only on the defect presence.

\subsection{Spectral indicator normalized by active power}

Spectral indicators have been largely used for the diagnosis of rolling element bearings in the steady regime. In the variable regime, these indicators change as the operating parameters change. The Fourier Transform (FT) of Equation (2) is as follows:

$$
X(f)=E_{i}(f) H(f)+B(f)
$$

with $E_{i}(f)=F T\left(\sum_{k=-\infty}^{\infty} F_{i}^{k} . \delta(n-k T)\right)$. We consider that in a short window of length $N$ the speed and the load are constant; under this condition the $F_{i}^{k}$ is constant and therefore $E_{N i}(f)=\frac{F_{i}}{T} \sum_{k=-\infty}^{\infty} \delta\left(f-\frac{k}{T}\right)$. We assume too that the impulse response $H(f)$ of the system does not change with speed. Equation (9) then becomes the following:

$$
X_{N}(f)=\frac{C_{1} v_{i} Q d e f}{T} \sum_{k=-\infty}^{\infty} . \delta\left(f-\frac{k}{T}\right) H(f)+B(f)
$$

It is seen from Equation (10) that $X_{N}(f)$ depends linearly to the rotation speed $v_{i}$, the load $Q$, and the size of the defect def. The proposed indicator consists in the normalization of the maximum of the FT, calculated on a window $N$, by the active power. It is given by the following equation:

$$
I=\max \left(X_{N}(f)\right) / P_{A}
$$




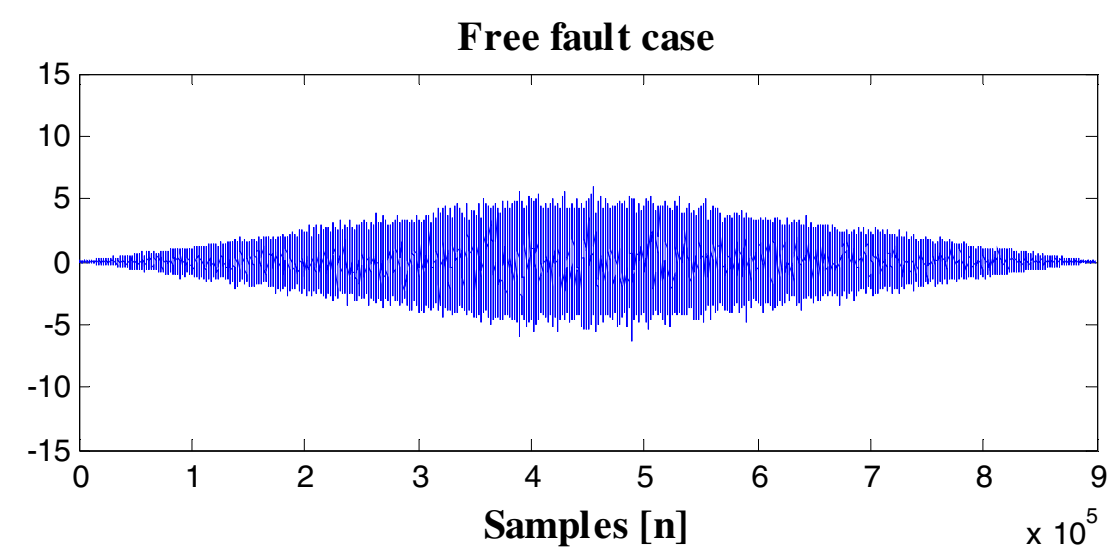

(a)

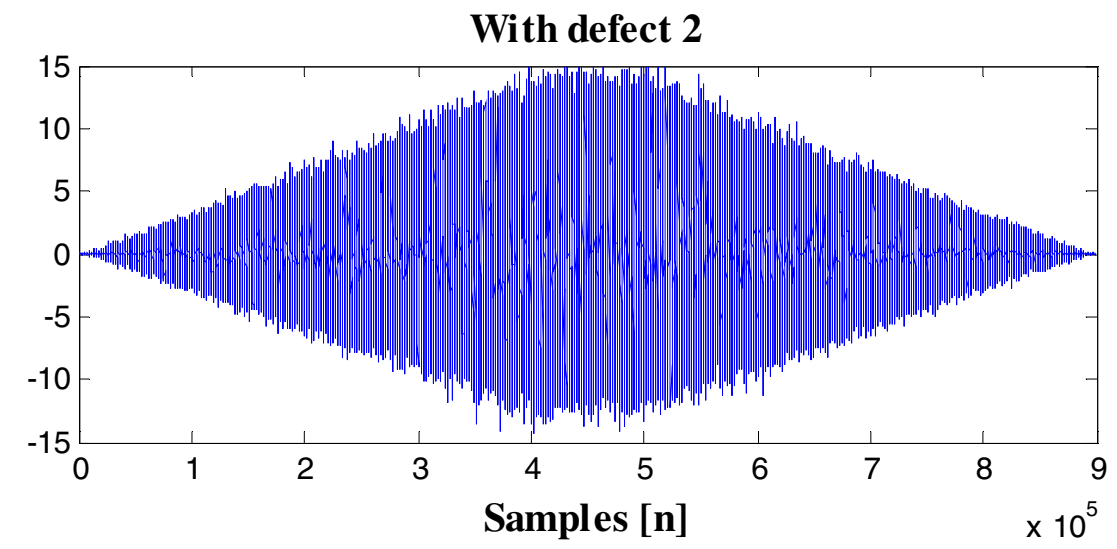

(b)

Fig. 2. (a) Typical signal in the free fault case 1. (b) Typical signal in the presence of defect 2.

This indicator is simple to implement and does not need large resolution. The algorithm to obtain this indicator is similar to the proposed algorithm for the $R M S$ value: the first step is acquiring the vibration signal at the same time as the current and voltage signals. Then for each segment of vibration signal and its corresponding voltage and current signals, we compute the FFT (using Hanning window) of the vibration segment and the active power, then divide the maximum of the obtained spectrum by the active power. This allows eliminating the influences of speed rotation and load from Equation (10). Therefore, the normalized spectral indicator $I$ is used for the diagnosis, which will depend only on the defect presence.

\section{Application to synthetic signals}

To test the proposed method, a simulation model of the vibration signal was used and is presented by the following equation:

$$
x(n)=\sum_{i} A_{i} h\left(n-i T-\tau_{i}\right)+b(n)
$$

with $h(n)$ as the impulse response generated by a single impact (assumed identical whatever the shape of the impact), $T$ is the time between two impacts (in variable regime, $T$ is related to rotating frequency), $A_{i}$ is the amplitude-modulation of the ith impact force, $\tau_{i}$ is the time lag from its time shock $i T$ due to the presence of slip, and $b(n)$ an additive background noise that represents the contribution of the other vibration sources. It is a simple model of a vibration signal produced by a single defect. This model has been successfully used to describe incipient faults in rolling element bearings [20,21]. For the simulation, only the normalized RMS value was tested.

For simulating active power, Equation (5) was used with the rotation speed $\Omega$ taking the profile as presented in Figure 5, and the parameters $T_{u}$ and $n$ are constants (for simplicity they are equal to one). For the simulation signals, three cases were considered: free fault, defects 1 and 2 . In the case of defect 2 , the modulation amplitude $A_{i}$ was more important than in defect 1 , whereas in the free fault case it was equal to zero. The sampling frequency was $10 \mathrm{kHz}$, the time of each signal was $90 \mathrm{~s}$, which gave $9 \times 10^{5}$ samples. Figure 2 a presents the signals produced by the Equation (12) in the case of free fault, and Figure $2 \mathrm{~b}$ in the presence of defect 2. It can be seen in these figures that simulation signals are modulated by rotation speed variation. In the faulty case, it can be seen 


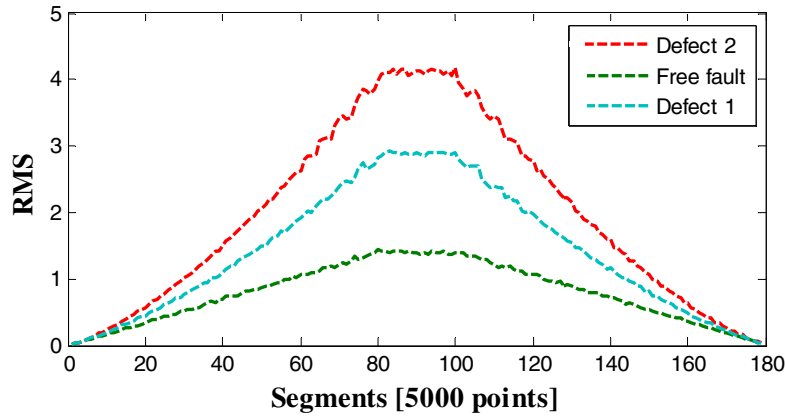

(a)

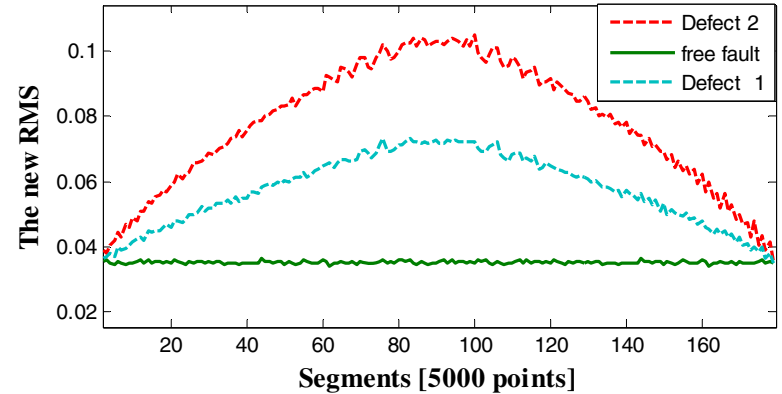

(b)

Fig. 3. (a) $R M S$ value for simulation signals for free fault and with fault (defects 1 and 2). (b) $R M S$ value for simulation signals after normalization by active power for free fault and with fault.

that the amplitude of signal is more important due to the presence of the defect.

The simulation signals were subdivided into segments (5000 points) and for each segment the $R M S$ value was computed and is presented in Figure 3a. The choice of 5000 points of the window size is empirical and it must respect that the signal is stationary in this segment. It can be seen in Figure 3a that speed variation has an impact on $R M S$ values. For example, the $R M S=1.2$ can indicate both free fault and faulty case.

Figure $3 \mathrm{~b}$ presents the normalized $R M S$ values by active power. In this figure, one can see that the new $R M S$ value in the free fault case is constant for all segments (each segment presents a given speed). When the fault is present, the normalized $R M S$ values begin rising and it can distinguish the faulty case from the free fault case, whatever the speed variation. The increase in normalized $R M S$ value is due to the presence of the impulse response generated by the impacts. It is noted that the new $R M S$ cannot classify the severity of the defect (distinction between defect 1 and 2) in a certain range of speed (low speed).

\section{Test rigs}

To test the proposed indicators on real signals, a series of experiments was performed to extract signals, in variable regime, on a test bench (Fig. 5a). This bench was equipped with a brushless motor with $10 \mathrm{~kW}$ power driven by an inverter, a module for the thrust bearings fatigue, a device for applying a load on the thrust bearings, and an oil injector for the lubrication. An ICP accelerometer sensor with a sensitivity of $9.87 \mathrm{mv} \cdot \mathrm{g}^{-1}$ is located on the axial direction of the thrust bearing. An incremental optical encoder, integrated into the motor, delivered 1024 point.rev ${ }^{-1}$. The optical encoder, which is a position sensor, allowed us to estimate the instantaneous speed. For the estimation of the active power, a current clamp was used to obtain the current and a voltmeter was used to obtain the electrical potential. For each test, the signals from the accelerometer, current clamp, voltmeter and the optical encoder were acquired simultaneously

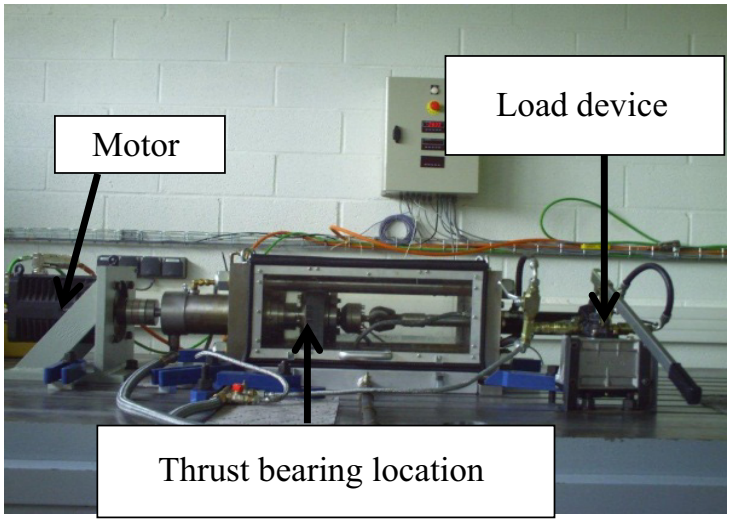

(a)

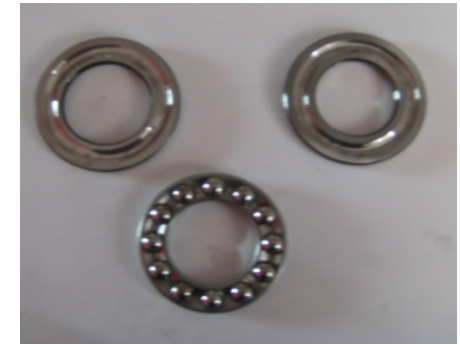

(b)

Fig. 4. (a) Test rigs. (b) Used thrust bearing.

by a dynamic acquisition system. For the accelerometer, current and voltage signals, the sampling frequency was $25600 \mathrm{~Hz}$; and for the optical encoder, the sampling frequency was $102400 \mathrm{~Hz}$. The acquisition time was $35 \mathrm{~s}$ (or 896000 samples for the vibration signal). Figure 4 shows the profile of the machine speed for all acquisitions: the speed rise area which is from 50 to $950 \mathrm{rpm}(15 \mathrm{~s})$, the speed constant area which speed is equal to $950 \mathrm{rpm}(5 \mathrm{~s})$, and the speed drop area which is from 950 to $50 \mathrm{rpm}$.

For the thrust bearings (see Fig. 5b), the spalling defects were created with different surfaces on one of its two rings. The surface of these defects was measured and is presented in Table 1. Figure 6 shows the microscopic 


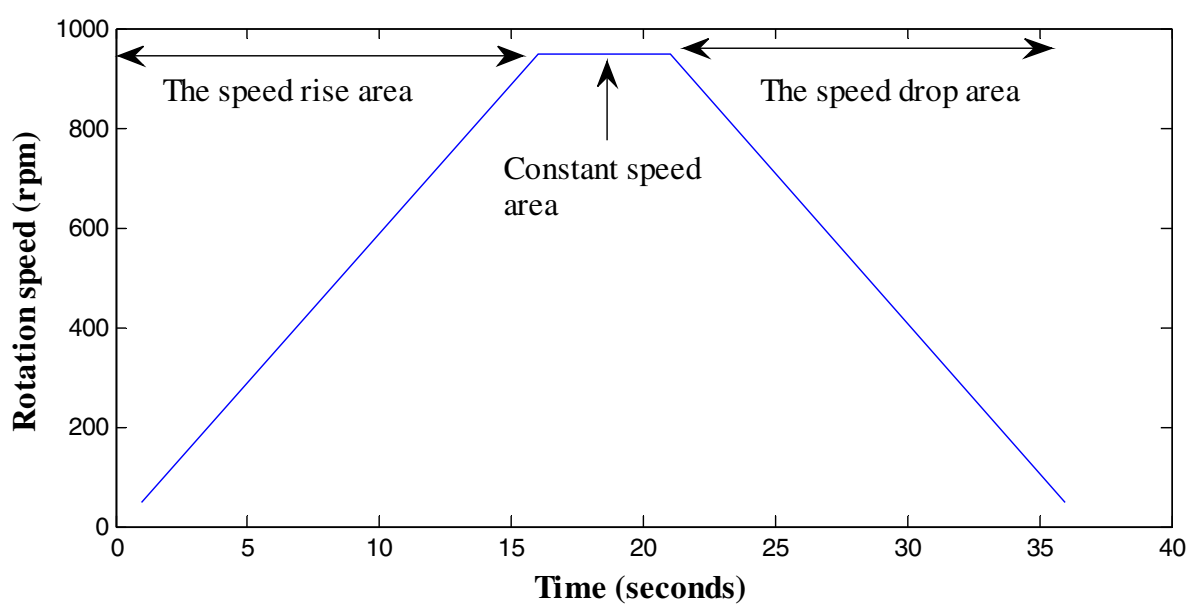

Fig. 5. Speed variation cycle.
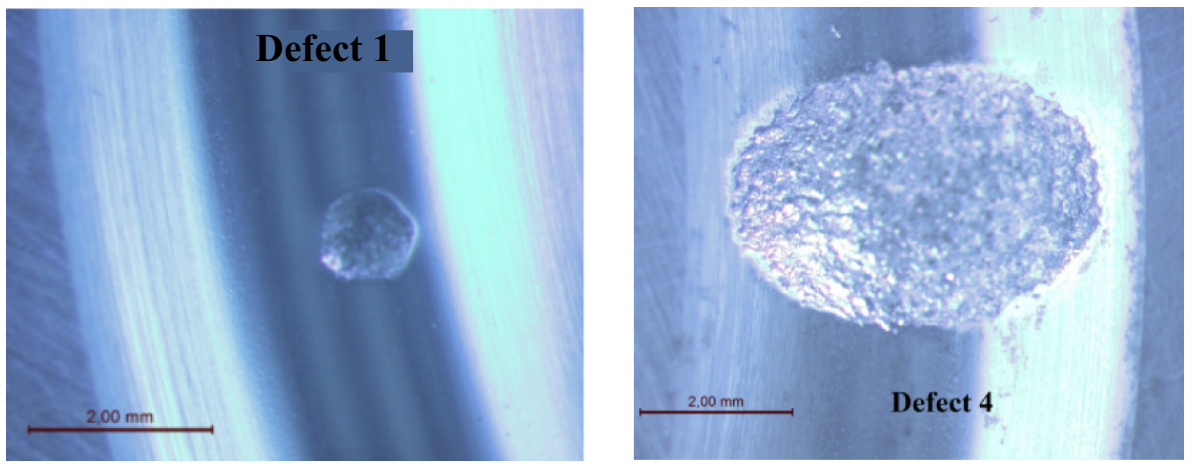

Fig. 6. Microscopic pictures of spalling defects 1 and 4 .

Table 1. The created spalling defect surfaces $\left(\mathrm{mm}^{2}\right)$.

\begin{tabular}{ccccc}
\hline $\begin{array}{c}\mathrm{N}^{\circ} \text { of } \\
\text { the defect }\end{array}$ & Defect 1 & Defect 2 & Defect 3 & Defect 4 \\
\hline $\begin{array}{c}\text { Surface } \\
\left(\mathrm{mm}^{2}\right)\end{array}$ & 0.8 & 3.6 & 9.2 & 14 \\
\hline
\end{tabular}

photos for defects 1 and 4 . Using the loading device, the following loads were applied to the thrust bearings for each degradation state: 0, 500, 1000, 1500, 2000, 2500 and $2900 \mathrm{daN}$.

\subsection{Signal analysis}

Figure 7 shows the vibration signals acquired in the cases of free fault and defect 4 for a load of 2900 daN. In the free fault case (Fig. 7a), we observed the progressive rise in vibration signal amplitude from $4 \mathrm{~m} . \mathrm{s}^{-2}$ to $18 \mathrm{~m} . \mathrm{s}^{-2}$ in the rising speed area, and in the drop speed area the amplitude decreased from $18 \mathrm{~m} . \mathrm{s}^{-2}$ to $4 \mathrm{~m} . \mathrm{s}^{-2}$. It can be seen that the amplitude of the signal is modulated by the speed variation. In the case of defect 4 , the signal amplitude increased from $4 \mathrm{~m} . \mathrm{s}^{-2}$ to $54 \mathrm{~m} . \mathrm{s}^{-2}$ in the speed rise area and decreased from $54 \mathrm{~m} . \mathrm{s}^{-2}$ to $4 \mathrm{~m} . \mathrm{s}^{-2}$ in the speed drop area (see Fig. 7b). It is obvious that the vibration signal amplitude is larger compared to the free fault case due to the presence of defect. In other words, it can be seen that the vibration signal amplitude is modulated by the variation of the speed and the presence of spalling defect.

Next, the signals acquired in the cases of free fault, defects 1-4 were segmented into slices of 5000 points, and on each slice the $R M S$ value was calculated and is presented in Figure 8a. This figure shows that the $R M S$ values in the areas where speed rises and drops follow a linear trend and these values depend strongly on the speed. The speed variation prevented us to put a fault detection threshold; for example, in this figure if the $R M S$ value equal to 4 is taken as a threshold, all the curves pass through this value and therefore the distinction between all cases is not possible using the classical $R M S$ value. It is shown in reference [15] that the $R M S$ value depends linearly on the speed. The trend of $R M S$ values as a function of active power is presented in Figure 8b (to avoid the overcrowding in this figure, we present just the following cases: free fault, defects 3 and 4 ). In this figure, the linear regression of the points formed by the $R M S$ values as a function of active power is presented. To test the quality of the regression, the coefficients of determination $r^{2}$ were calculated: $r^{2}=0.956$ in the free fault case, $r^{2}=0.935$ in the case of defect 3 , and $r^{2}=0.974$ in the case of defect 4 . We can see that these values are near to 1 , which demonstrate the fact that the $R M S$ values of the vibratory signal are 
K. Ait Sghir et al.: Mechanics \& Industry 17, 305 (2016)

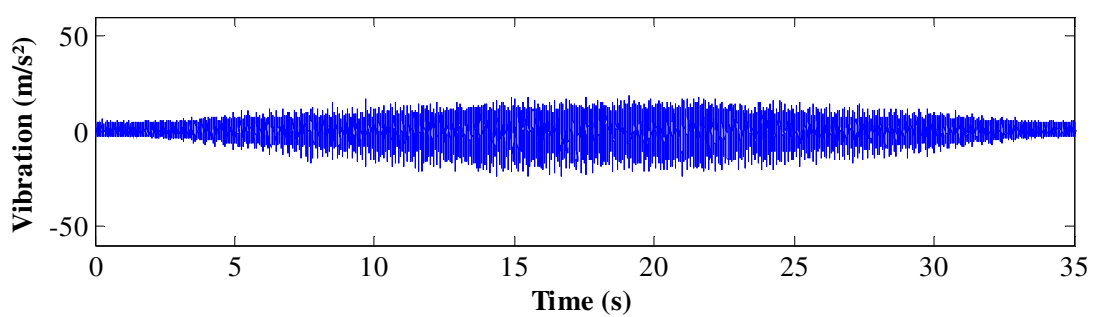

(a)

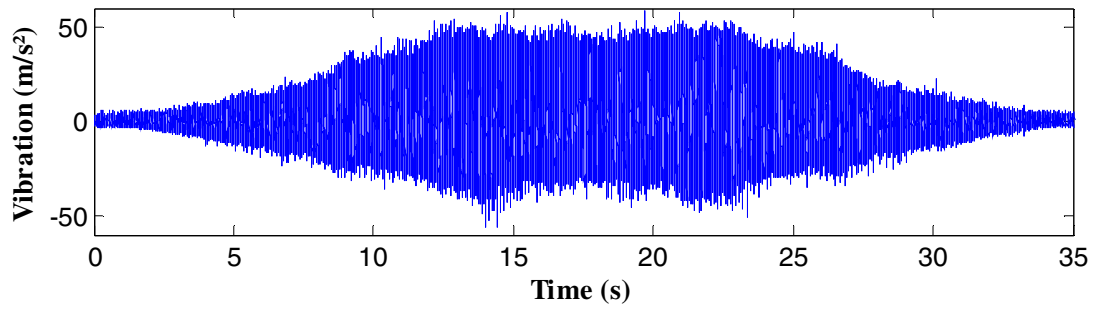

(b)

Fig. 7. (a) Typical signal recorded in the free fault case (load of 2900 daN). (b) Typical signal recorded in the case of defect 3 (load of $2900 \mathrm{daN}$ ).

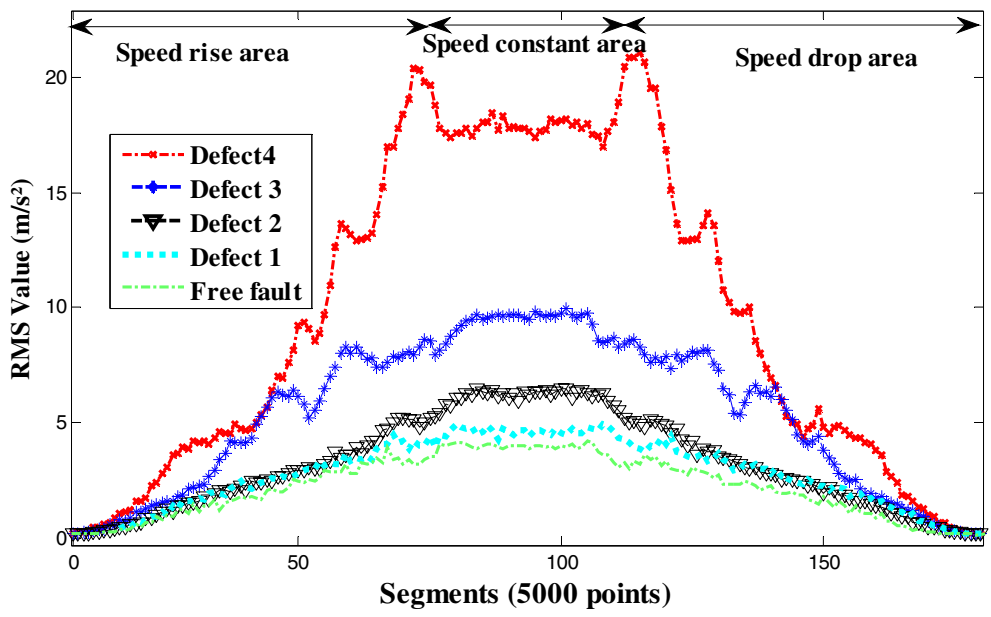

(a)

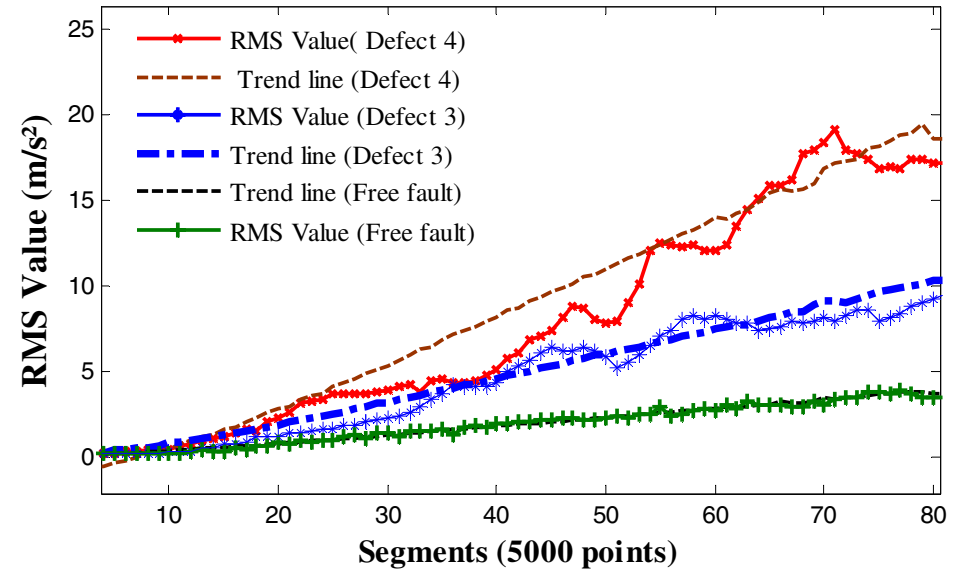

(b)

Fig. 8. (a) $R M S$ value depending on the thrust bearing states. (b) Linear regression of the $R M S$ values for different states in the speed rise area. 


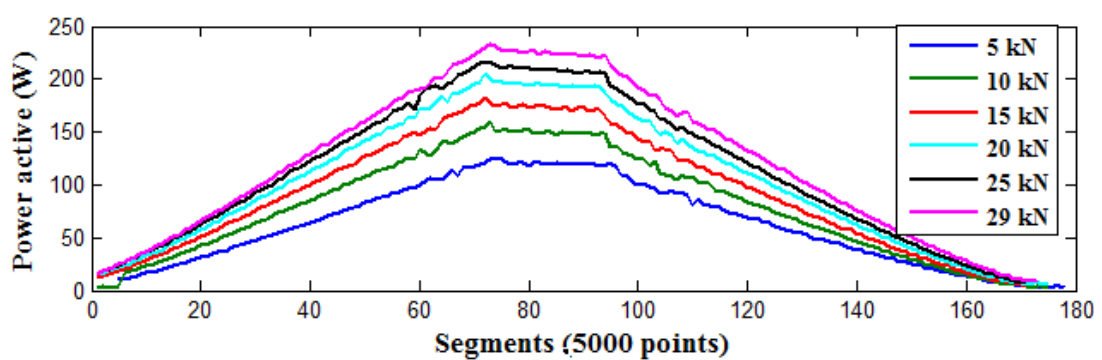

(a)

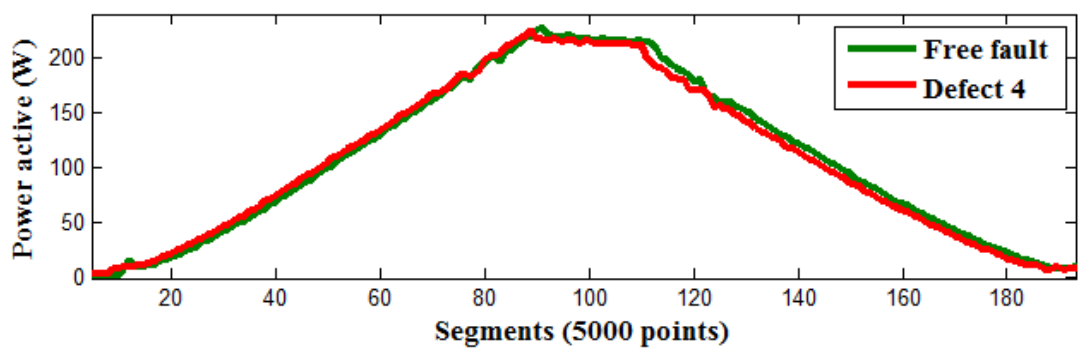

(b)

Fig. 9. (a) Active power for different loads. (b) Active power in the cases of free fault and defect 4.

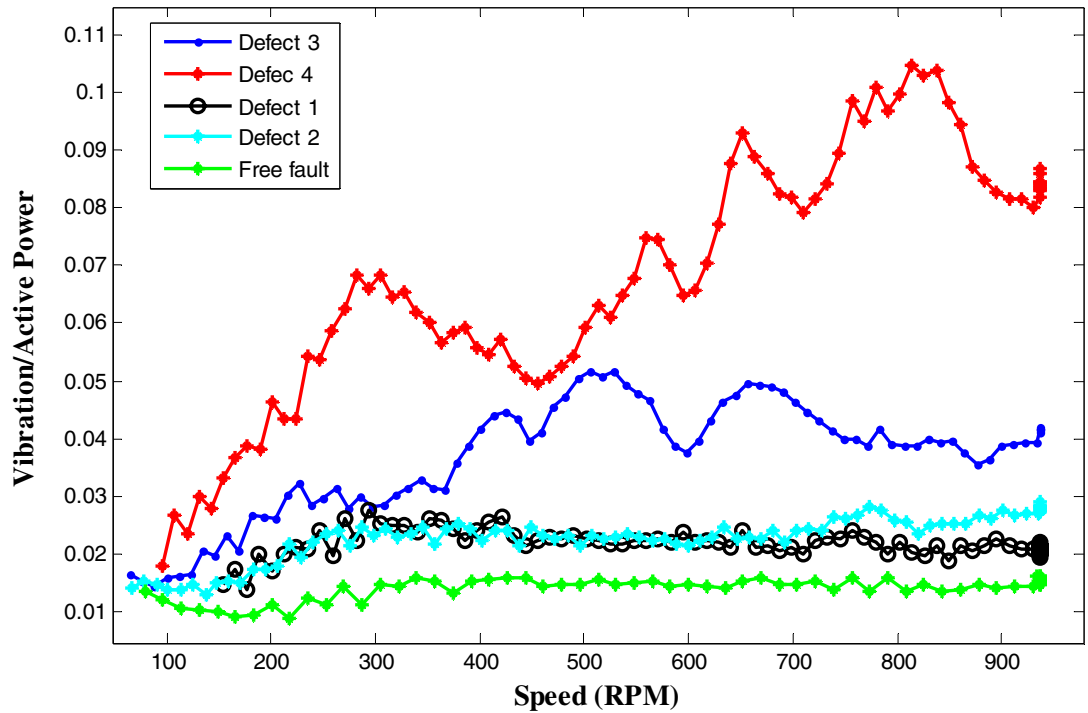

Fig. 10. Normalized $R M S$ value versus defects (load of 2900 daN).

a linear function of the active power. Figure 9a presents the active power estimated on each slice of 5000 points for loads of 5 to $29 \mathrm{kN}$ by steps of $5 \mathrm{kN}$. As we can see from this figure, the active power is a function of the speed and the load. Figure $9 \mathrm{~b}$ shows the active power in the cases of free fault and defect 4 . The active power is not affected by the defect presence; it depends only on the speed and the load. This result is in line with Equation (5). Figure 10 presents the normalized $R M S$ value by the active power for different states of thrust bearing degradation. As we can see from this figure, for the speed above $300 \mathrm{rpm}$ the normalized $R M S$ value has a stationary trend compared to classical RMS. Thus the normalization by active power eliminates the nonstationary appearance due to speed variation. The normalized $R M S$ value increases only according to the state of the thrust bearing degradation. The more important the defect is, the more the normalized corresponding indicator increases. It can be said that the normalized $R M S$ values increase with the size of the defect regardless of the speed variation. This is obvious for free fault, defect 1 and defect 2 . For defects 3 and 4 , there are some changes in their curves and compared with the classical $R M S$ it can be considered to have a stationary trend. Below $300 \mathrm{rpm}$, we can observe that speed has little influence on the new $R M S$ value, especially for defect 4 ; that is because the number of impulses due to the shock at windows size is small and the accelerometer response is weak at this speed range. It can be observed that for a speed less than $120 \mathrm{rpm}$, the new indicator is unable to distinguish between faulty and 


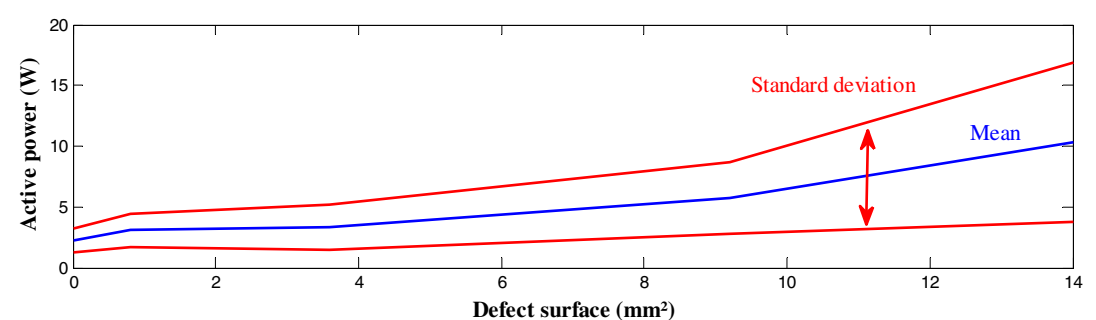

(a)

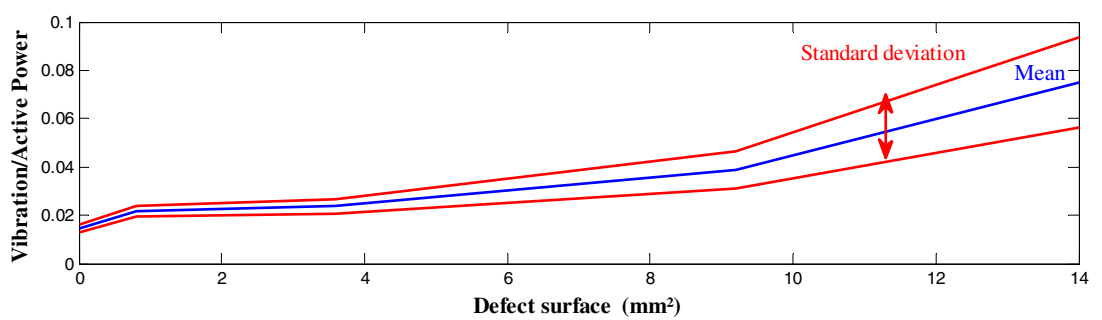

(b)

Fig. 11. Mean and dispersion of (a) classical $R M S$ of the signal vibration and (b) new $R M S$ value at a load of 2900 daN.

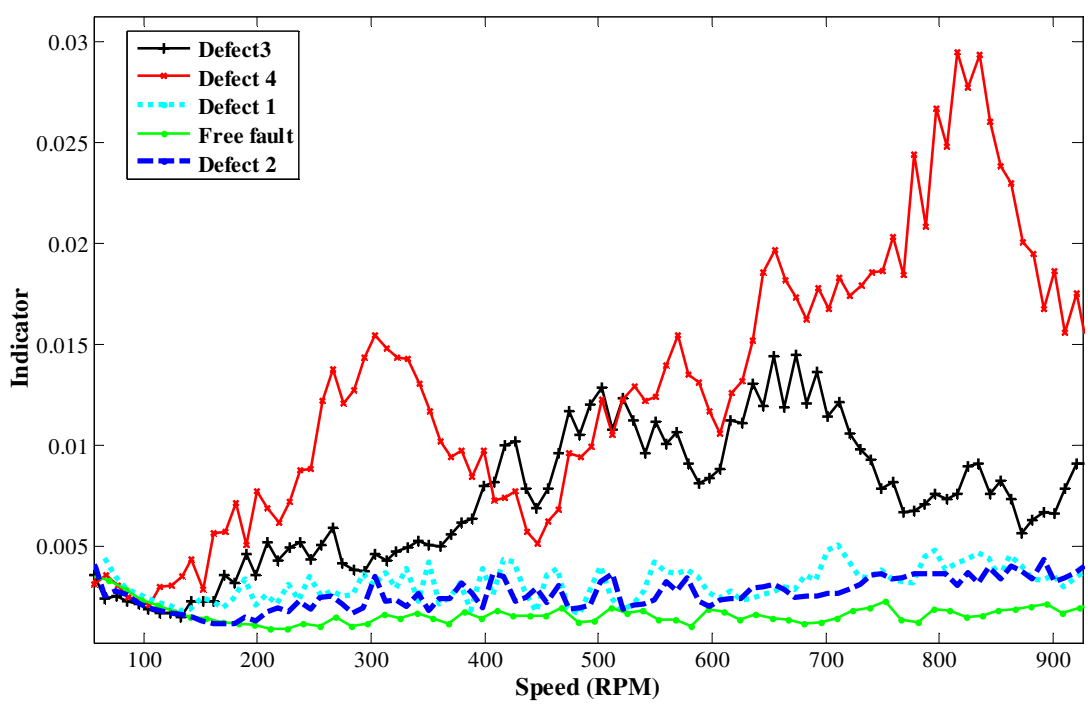

Fig. 12. Normalized spectral indicator versus defects (load of 2900 daN).

free fault cases. This is because at this speed range the accelerometer cannot react with the defect.

In the area corresponding to the speed rise between 150-and $950 \mathrm{rpm}$ (speed drop area is similar), the mean and standard deviation of the classical $R M S$ values (calculated for segments of 5000 points) were computed. Figure $11 \mathrm{a}$ presents the mean value and standard deviation for different states of degradation of the thrust bearings for a load of 2900 daN. It can be noted that the standard deviation is so large that if a threshold value is fixed to 3 for example, it indicates all bearing degradation states. This is due to the presence of the slope which greatly influences this classical indicator. Figure $11 \mathrm{~b}$ presents the mean value and standard deviation of the normalized $R M S$ (on slices of 5000 points) of the same vibration signals as in Figure 11a. It can be noted that the standard deviation around the mean became small. With this new indicator, one can distinguish the case without defect and with spalling defect. This classification can be done from a surface less than $2 \mathrm{~mm}^{2}$ in the case of a load of 2900 daN. This classification gives robustness to the new indicator in a variable regime.

\section{Normalized spectral indicator}

In the following, the indicator proposed in Section 2.4 was applied to the experimental signals for the segment size of 4096 points (the choice of this segment size is empirical and is taken as a power of 2 for FFT calculation). The results of the application for all degradation bearing states are presented in Figure 12. As can be seen from this figure, the indicator is correlated with the defect presence and is independent of the speed variation. For the free fault case, the indicator is stationary, the level of this indicator increased with the defect presence 
(defects 1 and 2). In the case of defects 3 and 4, the indicator increased very strongly. Due the weak response of the accelerometer at low speed, the indicator did not react with the defect under a speed of $240 \mathrm{rpm}$. To establish a diagnosis strategy, one can put a threshold value equal to this indicator in the case of defect 1 ; when the indicator exceeds this threshold, an alarm is triggered.

\section{Conclusion}

In this paper, the issue of bearing element monitoring at variable speed regime is addressed. The speed variation generates nonstationary components in the vibration signals, which affects strongly the classical tools and prevents use of these indicators for diagnostics.

Two indicators are proposed: $R M S$ value and a spectral indicator normalized by active power. An analytical model is given of the vibration signal as a function of speed load and defect size. The developed models justify the choice of those indicators to overcome the speed variation influence.

A test on the simulation signals is performed thanks to a simple model of the vibration signal producing a single defect. The results of simulation show clearly that in the free fault case, the new RMS is constant whatever the speed variation and when defect begins, the new $R M S$ rise depends only on the defect presence.

A test rig was prepared to collect vibration, tension and current signals for different states of the thrust bearing, which is necessary to test the proposed indicators. It was found that speed variation, load and defect presence modulate the vibration signals. The results show clearly that the proposed indicators are independent of speed variation influence at speeds above $300 \mathrm{rpm}$. The new indicators are able to diagnose the bearing state whatever the speed variation and it can also classify the severity of defect present, which is not achievable with classical $R M S$ value and spectral indicator. The new $R M S$ value at speeds below $300 \mathrm{rpm}$ depends slightly on the speed and it can distinguish between faulty and free fault cases.

The perspective of this work is to test those indicators on other kinds of bearings and to find a real application in the industrial domain.

Acknowledgements. This paper was financially supported by the "region de Champagne-Ardenne" and European funds FEDER.

\section{References}

[1] B.T. Kuhnell, Wear in rolling element bearings and gears-how age and contamination affect them, Machinery Lubrication Magazine, Monash University, 2004

[2] N. Tandon, B.C. Nakra, Detection of defects in rolling element bearings by vibration monitoring, J. Instit. Eng. 73 (1993) 271-282
[3] R.A. Collacott, Mechanical fault diagnosis, Chapman and Hall, London, 1977

[4] K.S. Jardine, D. Lin, D. Banjevic, A review on machinery diagnostics and prognostics implementing conditionbased maintenance, Mech. Syst. Signal Process. 20 (2006) $1483-1510$

[5] N. Tandon, A. Choudury, A review of vibration and acoustic measurement methods for the detection of defects in rolling element bearings, Tribol. Int. 32 (1999) 469-480

[6] P.D. McFadden, J.D. Smith, Vibration monitoring of rolling element bearings by the high frequency resonance technique - a review, Tribol. Int. 17 (1984) 3-10

[7] P.D. McFadden, J.D. Smith, Model for the vibration produced by a single point defect in a rolling element bearing, J. Sound Vib. 96 (1984) 69-82

[8] P.D. McFadden, J.D. Smith, The vibration produced by multiple point defects in a rolling element bearing, J. Sound Vib. 98 (1985) 263-273

[9] G. Dong, J. Chen, Noise resistant time frequency analysis and application in fault diagnosis of rolling element bearings, Mech. Syst. Signal Process. 33 (2012) 212-236

[10] R.B. Randall, J. Antoni, Rolling element bearing diagnostics-A tutorial, Mech. Syst. Signal Process. 25 (2011) 485-520

[11] J. Antoni, Cyclic spectral analysis in practice, Mech. Syst. Signal Process. 21 (2007) 597-630

[12] W. Bartelmus, R. Zimroz, A new feature for monitoring the condition of gearboxes in non-stationary operating conditions, Mech. Syst. Signal Process. 23 (2009) 15281534

[13] H. Wang, P. Chen, Fuzzy diagnosis method for rotating machinery in variable rotating Speed, IEEE Sensors Journal 11 (2011) 23-34

[14] J. McBain, M. Timusk, Fault detection in variable speed machinery: Statistical parameterization, J. Sound Vib. 237 (2009) 623-646

[15] K. Ait Sghir, F. Bolaers, O. Cousinard, J.P. Dron, Vibratory monitoring of a spalling bearing defect in variable speed regime, Mechanics \& Industry 14 (2013) 129136

[16] L.F. Villa, A. Renones, J.R. Perana, J.L. De Miguel, Statistical fault diagnosis based on vibration analysis for gear test-bench under non-stationary conditions of speed and load, Mech. Syst. Signal Process. 29 (2012) 436-446

[17] N. Tandon, A. Choudury, An analytical model for the prediction of the vibration response of rolling element bearings due to localized defect, J. Sound Vib. 205 (1997) 275-292

[18] C. Zhang, Defect detection and life prediction of rolling element bearings, Thesis, Georgia Institute of Technology, 2001, Chap. V, pp. 97-123

[19] F. Bolaers, S. Rémond, X. Chiementin, S. Crequey, J.P. Dron, Modélisation de la force d'impact due a un écaillage de fatigue dans les roulements, Premier Colloque International IMPACT 2010, Djerba, Tunisie, 2010, pp. 22-24

[20] J. Antoni, R.B. Randall, Differential diagnosis of gear and bearing faults, ASME J. Vib. Acoust. 124 (2002) 165-171

[21] J. Antoni, R.B. Randall, The spectral kurtosis: a useful tool for characterizing non-stationary signals, Mech. Syst. Signal Process. 20 (2006) 282-307 